\title{
Development and Evaluation of Online Approaches for Improved Kinaesthetic Learning in Science
}

\author{
Anna M. Scanlan ${ }^{1,2}$, Declan Kennedy ${ }^{2}$, Tommie V. McCarthy ${ }^{1}$ \\ ${ }^{1}$ School of Biochemistry and Cell Biology, University College Cork, Cork, Ireland, ${ }^{2}$ School \\ of Education, University College Cork, Cork, Ireland.
}

\begin{abstract}
Kinaesthetic learning is expressed when physical actions are used to connect concept development to reality, for example through model building, trial and error practice, or role-play interactions. Learning through a kinaesthetic modality is highly effective and complementary to other learning modalities. Recent advances in gamification for education have increased access to science simulations and learning online. However, the transfer of offline kinaesthetic techniques to online learning remains under-researched and poorly implemented on affordable, scalable platforms. Here we describe an accessible approach for educators on how to incorporate online kinaesthetic aspects into lessons through use of a scalable and affordable framework developed called the 'Kinaesthetic Learning System' (KLS). This framework should be of particular use for learning complex molecular life science topics but can be adapted and modified independently by the educator to address different knowledge levels and for expansion to other disciplines.
\end{abstract}

Keywords: Kinaesthetic; e-learning; education; online-learning; biochemistry; life science. 


\section{Introduction}

The human brain is an extraordinarily complex organ that employs multiple cortical structures to process, store and integrate information, through multiple sensory systems (Kolb \& Whishaw, 1996). When multiple areas of the brain are engaged by a task, learning is significantly increased by comparison with learning through one sensory modality such as reading alone or listening alone (Mayer, 2003). Digital technology potentially offers significant advantages over traditional learning approaches as it has the capability to provide increased opportunities for better engagement of multiple cognitive centres of the brain. However, the majority of third level education (University level) content on digital platforms is based on visual and auditory delivery but typically neglects kinaesthetic aspects. The latter offers an approach to engage additional sensory neural elements and thus enhances the efficacy of learning.

Kinaesthetic learning $(\mathrm{KL})$ or tactile learning occurs when a person uses physical actions to connect concept development to reality, such as through the medium of model building, trial and error practice or role-play interactions. As such, it is easier to recognise kinaesthetic learning rather than define it, but an apt definition is provided by Balasubramaniam and Indhu (2016) which states that "Kinaesthetic (K) learners prefer to learn by connecting to reality and they acquire information through experience and practice." (p.16)

$\mathrm{KL}$ is significantly under-developed for third level online learning in comparison to other learning modalities, namely reading/writing (RW), visual (V) and auditory (A). The major advances in software, hardware and cloud computing systems in recent years has made the prospect of developing online education material enhanced through the application of KL increasingly feasible. The learning of molecular sciences at third level is particularly challenging due to new concept density and the sub-microscopic nature of molecules which makes visualisation very difficult. Consequently, the learning of molecular sciences stand to gain significantly through kinaesthetic approaches.

\section{The case for online kinaesthetic learning based on neuroscience}

Studies on basic nervous systems have demonstrated how multiple parts of the neural system are capable of learning (Kolb \&Whishaw, 1996; Bedecarrats, Chen, Pearce, Cai, Glanzman, 2018). This infers that the different neural areas are capable of different neural memories. The visual system, for example, can retain memory for colour, shape, and movement. Kolb and Wishaw outline how neural areas can be further subdivided for memory and interlinked with different regions of the brain to process incoming information from multiple stimuli. With the brain's capacity to collect information and store it in such a variety of memory formats, it becomes apparent why learning increases when multiple regions of the brain are engaged in a learning task. 
In systemic terms, information from the body and external environment is carried to the brain along sensory axons emanating from sensory organs such as the ears, eyes, and skin. Motor nerves controlling all bodily movement run from the motor cortex to the rest of the body in the opposite direction of sensory axons (Kolb \& Whishaw, 1996). From an evolutionary standpoint, this integration of physical and cognitive neural structures, as activated during $\mathrm{KL}$, allows a more efficient means of processing information. For example, consider how much easier it is to demonstrate how to undo a zip compared with having to write out a stepby-step guide or give a detailed oral account of the action. A kinaesthetic approach to online learning that mimics the physical act of demonstrating how something works, has the potential to successfully integrate KL and digital technology for enhanced education.

A final factor to support KL is to understand the role of the cerebellum, one of the oldest regions of the brain in evolutionary terms. The cerebellum is responsible for procedural memory and motor control, and co-ordinates fine movements with learning (Crossman \& Neary, 1995). The cerebellum links learning and movement in a way that may be accessed through KL approaches that are physical and practiced during a learning task and may contribute to making learning more intuitive.

\section{Designing a kinaesthetic approach to online learning}

Research on kinaesthetic approaches to online learning is sparse and has been limited by the lack of technological platforms. Digital technologies are now sufficiently advanced to support kinaesthetic approaches. However, development and optimisation of KL is necessary to validate and underpin its widespread adoption. Design of the individual elements suitable for incorporation into KL approaches should draw from the well-established work of Mayer and others who have identified and validated instructional guidelines for optimal online learning. The key aspects of these guidelines are outlined in Table 1 below. 
Table 1. E-learning guidelines for the development of online lessons

\begin{tabular}{|c|c|}
\hline Guidelines & Authors \\
\hline $\begin{array}{l}\text { Spatial contiguity effect: } \\
\text { Position relevant text beside informative pictures }\end{array}$ & (Clark \& Mayer, 2008) \\
\hline $\begin{array}{l}\text { Multimedia effect: } \\
\text { Present lessons in a multimedia format }\end{array}$ & $\begin{array}{l}\text { (Mayer,2003; Sankey, Birch, Gardiner, } \\
\text { 2011) }\end{array}$ \\
\hline $\begin{array}{l}\text { Coherence effect: } \\
\text { Keep content specific with no extraneous details }\end{array}$ & (Mayer, 2003) \\
\hline $\begin{array}{l}\text { Personalisation effect: } \\
\text { Use conversational language rather than formal }\end{array}$ & (Mayer, 2003) \\
\hline $\begin{array}{l}\text { Perspective Principal: } \\
\text { View online lessons from } 1 \text { st person perspective }\end{array}$ & (Fiorella et al., 2017) \\
\hline $\begin{array}{l}\text { Segmenting Principal: } \\
\text { Facilitate paced learning \& design navigational controls }\end{array}$ & (Clark \& Mayer, 2008) \\
\hline $\begin{array}{l}\text { Deliver audio with diagrams or animation but not with } \\
\text { both text and diagrams }\end{array}$ & (Clark \& Mayer, 2008) \\
\hline $\begin{array}{l}\text { Facilitate interactions with online learning content } \& \text { tes } \\
\text { with difficult concepts }\end{array}$ & $\begin{array}{l}\text { st (Gegner, Mackay, Mayer, 2009; Saadé, } \\
\text { Morin, Thomas, 2012) }\end{array}$ \\
\hline Provide dual cues for recall e.g., visual with auditory & (Xie, Mayer, Wang, Zhou, 2018) \\
\hline
\end{tabular}

\subsection{Defining the framework}

A methodical effort was applied toward developing an accessible kinaesthetic learning system suitable for online platforms. A set of criteria was generated to guide development of such a system and is outlined in Table 2 . 
Table 2. Criteria for development of a Kinaesthetic Learning System (KLS) for online platforms

\section{List of Criteria for a KLS}

User friendly, affordable, adaptable, scalable, and expandable

Simple design that lends itself to reproduction in accessible formats

High engagement to maintain interest and attention

Facilitate assessment

Facilitate long-term learning

Present incremental challenges to the learner

Amenable to the application of scientific research methodology i.e., design, build, test, repeat

Be a true translation of traditional kinaesthetic learning approaches

Be amenable to measurable evaluation

There are many potential platforms that could support kinaesthetic approaches. Presentation software programmes typically have many features that could support KL including mouse or touchpad directed movement of objects, read/write, visual, audio and animation aspects. PowerPoint is a well-developed and widely used programme in this category and is highly accessible across education sectors. Thus, it can be considered a suitable platform for exploring kinaesthetic based lesson development.

\subsection{Exploring Kinaesthetic Learning using a Complex Topic in Molecular Science}

The Lac Operon in E. coli is a paradigm for gene regulation and is a complex and well elucidated genetic system and a typical core element of the molecular life sciences curriculum. The Lac Operon lesson is a conceptually difficult and challenging science topic and was chosen as a test case to explore development of approaches that meet the criteria outlined (Table 2).

Narrative for the Lac Operon lesson was developed for KL and various layouts of text and diagram elements were explored. The topic was initially broken down into stepwise segments covering over 40 concepts and reconstructed into a stepwise narrative to facilitate KL. Over 30 illustrations were created, with each illustration representing an individual element of the operon. Each element was imported into PowerPoint as icons that the students could move about independently onscreen and within PowerPoint slides, through use of a mouse, tracker pad or touchscreen. This facilitated the construction of different snapshots representing key gene regulatory events in the operation of the Lac Operon. The generation of multiple, independent icons also facilitated creation of a full animated version of the Lac Operon (link to animation: https://youtu.be/Mti9I7fDKwc) and enabled the building of different scenario diagrams for the operon that could be assembled and disassembled in a kinaesthetic manner. 
The independent icon aspect of the design facilitates numerous kinaesthetic approaches compatible with PowerPoint. Two approaches that utilised a "guided assembly" and an "unguided assembly" were selected for development with the lesson and several iterations of these approaches were examined. The final iteration of the "guided assembly" approach chosen involved providing a boxed set of icons in random order at the top of a PowerPoint slide for a given Lac Operon scenario. A diagram outline of the scenario was provided in the lower part of the slide with placeholder prompts for specific icons. This enabled implementation of the kinaesthetic aspect by clicking and dragging the relevant icons from the box into position over the correct placeholder.

The "unguided assembly" involved provision of a boxed set of icons for a Lac Operon scenario enabling implementation of the kinaesthetic aspect by assembling a diagram of the scenario in the space below the box but without the aid of placeholder prompts, according to a set of instructions. Essentially, both conditions enable a student to work independently in PowerPoint and to interact with the Lac Operon lesson by reassembling various scenarios at their own pace. This design facilitated the building of multiple levels of difficulty by altering the level of prompts/instructions provided and/or the range of icons deposited in the box. This overall design is directly applicable to the numerous molecular and biochemical process that are central to the molecular life sciences. Moreover, although not covered here, it can also be easily adapted to offer an alternative to traditional forms of assessment.

Feasibility testing of this format with a cohort of 40 undergraduate medical students was carried out using an early iteration of the KL PowerPoint lesson containing features as outlined in Section 3.2, paragraph 2, but which did not include a video aspect. The layout of the PowerPoint included introductory instruction slides on how to navigate and interact with the lesson. Lesson notes appeared on the left of each slide with descriptive illustrations matching the text on the right. The wordcount for the main text body of the lesson, excluding introductory slides, headings and diagram labels was approximately 1,700 and was split across 7 slides. This represents a complete one hour foundational third level science lesson on the Lac Operon and as such, is more suitably realistic for developing and testing the KLS.

Each static lesson slide was followed by an interactive task slide (7 in total) containing identical text and illustrations from the previous slide. Illustrations on the task slide were moveable for guided assembly through use of a tracker pad, mouse, or touchscreen. It was observed that students who used a mouse found the interaction easier than those who used a tracker pad or touchscreen, but all students were able to complete the study and assembly tasks within the allotted time frame of 25 minutes. Some students participated on their mobile phones but most participated on laptops. Students using PCs reported less technical difficulties in navigating PowerPoint than those using Mac devices. Key feedback improvements taken on board from the student cohort led to the following improvements in the KLS: 
- Provision of improved instructions for navigating the lesson

- $\quad$ Provision of detailed stepwise information on how to use PowerPoint (as the level of PowerPoint knowledge varied)

- Reduction in the complexity of diagrams by reducing schematic content in favour of illustrative

- Incorporation of a video made from animated lesson icons and lesson notes to provide an overview of the lesson prior to study

\section{Discussion and Conclusion}

The molecular sciences are dense with theories and abstract concepts that cannot be readily visualised. Consequently, learning of the molecular sciences is particularly challenging by comparison with subjects which can be readily visualised. For example, anatomy contains familiar concepts (e.g., digestion) which can be explained using relatable visuals and familiar terminology (e.g., cells). Contrast this with the molecular life sciences replete with unfamiliar concepts (e.g., ionotropic receptor activation), which are generally represented as schematic visuals with basic shapes, intertwined with arrows and taught using alien terminology (e.g., cyclic adenosine monophosphate). Molecular science learning is hampered by the common use of abstract language and visuals to explain abstract processes and accordingly, reduces the relatability of the subject matter. KLS is a framework with the potential to address the challenge of making molecular science more relatable and tangible for learning.

Students can use the visual and interactive aspect of KL assembly to engage in various lesson scenarios, study them repeatedly if they so choose and at their own pace, in a manner that supports intrinsic processing (Mayer RE, Hegarty M, Mayer S, Campbell J, 2005) while minimising cognitive overload. This addresses one of the major advantages that static, paperbased learning has retained over computer-based learning thus far. Students using the KL assembly approach in PowerPoint do not require prior knowledge of the lesson content as they can interact with onscreen content in a stepwise manner in tandem with learning as they proceed through the slides. This enables a faster process of knowledge attainment for the student and reduces the time an educator must spend introducing the background information for a topic. A key aspect of the KL assembly design is that it facilitates the rapid assessment of student engagement, as judged by the level of correctly assembled scenarios, prior to examining students via traditional assessment formats.

Several successful translations of kinaesthetic approaches to online learning do exist in the form of simulations for laboratory science (for example, the 'Anatomage Table' (https://www.anatomage.com/table/). However, the majority of these simulations do not meet the KLS criteria outlined, particularly with respect to adaptability, affordability, simplicity in 
design and user directed expandability. The KLS outlined here offers a framework which educators can use to develop, adapt, and build bespoke KL assembly approaches for enhancing their lesson material and to customise these to match the level and ability of their learner cohorts.

In conclusion, our work demonstrates how the format developed is feasible for integrating a KL approach into complex molecular life sciences. Adopting, evolving, and evaluating these approaches will enhance their efficacy going forward.

\section{References}

Balasubramaniam G, Indhu K. 2016. A study of learning style preferences among first year undergraduate medical students using VARK model. Education in Medicine Journal. 8(4):15-21. doi: 10.5959/eimj.v8i4.440

Bedecarrats A, Chen S, Pearce K, Cai D, Glanzman DL (2018) RNA from trained aplysia can induce an epigenetic engram for long-term sensitization in untrained aplysia. eNeuro 5.0038-18.2018; doi: https://doi.org/10.1523/ENEURO.0038-18.2018

Clark RC, Mayer RE (2008) E-Learning and the science of instruction: proven guidelines for consumers and designers of multimedia learning, 2nd Edition. San Francisco, Calif.: Pfeiffer. ISBN-13: 9780787986834

Crossman, A. R., \& Neary, D. (1995). Neuroanatomy: An illustrated colour text. Edinburgh: Churchill Livingstone.

Fiorella L, van Gog T, Hoogerheide V, Mayer RE (2017) It's all a matter of perspective: Viewing first-person video modeling examples promotes learning of an assembly task. Journal of Educational Psychology 109:653-665. http://dx.doi.org/10.1037/edu0000161

Gegner JA, Mackay DHJ, Mayer RE (2009) Computer-supported aids to making sense of scientific articles: cognitive, motivational, and attitudinal effects. Educational Technology Research \& Development 57:79-97. doi 10.1007/s11423-008-9088-3

Kolb B, Whishaw IQ (1996) Fundamentals of Human Neuropsychology, 4th Edition. New York, United States of America: W.H. Freeman and Company. ISBN: 2900716795864

Mayer RE (2003) The promise of multimedia learning: using the same instructional design methods across different media. Learning \& Instruction 13:125. Publisher: Elsevier Science; [Journal Article], Database: APA PsycInfo. Retrieved from https://www.ebsco.com/

Mayer RE, Hegarty M, Mayer S, Campbell J (2005) When static media promote active learning: annotated illustrations versus narrated animations in multimedia instruction. Journal of Experimental Psychology Applied 11:256-265. doi: 10.1037/1076898X.11.4.256

Saadé RG, Morin D, Thomas JDE (2012) Critical thinking in e-learning environments. Computers in Human Behavior 28:1608-1617. Publisher: Elsevier Science; [Journal Article], Database: APA PsycInfo.. etrieved from https://www.ebsco.com/

Sankey MD, Birch D, Gardiner MW (2011) The impact of multiple representations of content using multimedia on learning outcomes across learning styles and modal preferences. International Journal of Education \& Development using Information \& Communication 
Technology 7:18-35. Database: Education Full Text (H.W. Wilson). Retrieved from https://www.ebsco.com/

Xie, H., Mayer, R. E., Wang, F., \& Zhou, Z. (2018, July 2). coordinating visual and auditory cueing in multimedia learning. Journal of Educational Psychology. Advance online publication. http://dx.doi.org/10.1037/edu0000285 